\title{
On the other hand: including left- handers in cognitive neuroscience and neurogenetics
}

\section{Roel M. Willems, Lise Van der Haegen, Simon E. Fisher and Clyde Francks}

\begin{abstract}
Left-handers are often excluded from study cohorts in neuroscience and neurogenetics in order to reduce variance in the data. However, recent investigations have shown that the inclusion or targeted recruitment of left-handers can be informative in studies on a range of topics, such as cerebral lateralization and the genetic underpinning of asymmetrical brain development. Left-handed individuals represent a substantial portion of the human population and therefore left-handedness falls within the normal range of human diversity; thus, it is important to account for this variation in our understanding of brain functioning. We call for neuroscientists and neurogeneticists to recognize the potential of studying this often-discarded group of research subjects.
\end{abstract}

Roughly $10 \%$ of humans prefer to use their left hand for manual actions such as writing, brushing teeth and using scissors. The minority status of left-handers, combined with abundant left-right symbolism across cultures, has intrigued people in different civilizations, from ancient times until the present day ${ }^{1,2}$. This has resulted not only in stigma $^{3}$ but also in equally unfounded claims concerning left-handers' exceptional abilities as reported by the popular media every year around 13 August (International Left-Handers Day). Rather than focusing on the mysterious nature of this trait, this Opinion article argues that left-handers are a compelling and widely available but largely untapped resource for neuroscience and neurogenetics studies.

In this article, we first summarize how left-handedness is defined, noting some key points regarding its evolution and development. We then explain why left-handers are often barred from being experimental subjects and argue that this exclusion is mostly unnecessary. We show how several recent studies of embodied cognition, cerebral lateralization and behavioural genetics have in fact benefited from the inclusion or targeting of left-handed participants. Last, we propose some ways in which left-handedness can be studied to address additional questions in cognitive neuroscience and neurogenetics.

\section{Defining left-handedness}

On the face of it, categorizing people as left-handed or right-handed seems simple enough. For example, the widely used
Edinburgh Handedness Inventory, which is often administered as a self-report questionnaire, assesses hand preference for a range of common manual tasks ${ }^{4}$. The inventory includes an indication of the strength of hand preference for each task and can be updated to include only items that are culturally relevant to a particular target population ${ }^{5,6}$. In the general population, the resulting laterality index $-\mathrm{a}$ measure of the direction and strength of preference across all items - is bimodal ${ }^{4}$; in other words, the majority of subjects score towards one or the other extreme of the distribution for left- or right-handedness. There are of course people who do not demonstrate a clear-cut overall hand preference, and they can variously be categorized as right-handed, ambidextrous or left-handed according to their score on the laterality index. Although the imposition of a small number of categories on the continuous laterality index necessarily involves arbitrary cut-off points, this does not invalidate the dichotomous interpretation of hand preference for the majority of people that is apparent in the population distribution from this kind of inventory. Many additional ways of measuring and scoring hand preference - too numerous to review here - have been devised and debated. A general consensus is that because hand preference can vary by task, assessments based on multiple tasks are preferable to those based on a single task (for example, just asking about a subject's writing hand) and to assessments that are made without reference to any specific task (for example, simply asking 'are you left- or right-handed?').

Motor performance tests, such as pegmoving or finger-tapping tasks, provide quantitative measures related to handedness ${ }^{7-10}$. Many interesting associations between handedness and diverse aspects of cognition, behaviour and even personality have been revealed through the use of such quantitative indices or through categorizing mixed-handers as an intermediate, third group ${ }^{11-15}$. In addition, some recent molecular genetics studies have provided insights into handedness that was assessed using quantitative performance indices (see below). However, the exclusion criterion of left-handedness that is so often applied in neuroscience studies is usually based on questionnaire-defined hand preference rather than on performance-based indices.

Hand preference is strongly - although not perfectly - correlated with foot preference, which is also strongly lateralized towards the right in humans at a population level ${ }^{16,17}$. Although left-footedness may be more closely linked to a reorganization of certain cortical functions than left-handedness ${ }^{18}$, it is not used as an exclusion criterion in neuroscience studies and has not been as extensively studied.

\section{Origins of handedness}

A strong population-level bias towards the right hand has probably existed in the human lineage as far back in time as it is possible to infer, including from the orientation of marks or strokes left by the manufacturers of stone tools and cave paintings ${ }^{19,20}$. This bias is present in all world populations that have been studied ${ }^{19}$, although the rate of left-handedness may vary within a range of $4-16 \%$, possibly for biological, cultural or assessment reasons ${ }^{21}$. Remarkably, the population-level bias towards righthandedness is already apparent very early in human development. Ultrasound scanning has shown that at 10 weeks of gestation, $85 \%$ of human foetuses move their right arms more than their left arms $\mathrm{s}^{22}$ and that early fetal motor asymmetry is a stable predictor of handedness later in life ${ }^{23}$. Together, these observations suggest that the predominance of right-handedness in our species has a genetic basis and is likely to be just one manifestation of a broadly lateralized programme of motor and cognitive development ${ }^{24}$. Leftright differences in brain morphology can be detected by ultrasound scanning as early as 20 weeks of gestation in the human foetus ${ }^{25}$. 
In fact, lateralization of CNS structure and function is found across vertebrates ${ }^{26}$ and probably reflects the general, dual advantages of efficiency of function within each of the two sides and division of labour between them. This suggests that some developmental mechanisms underlying human handedness and CNS asymmetry are likely to be very ancient, even if other aspects of the elaboration of asymmetry in the human brain, such as its lateralization for language, are evolutionarily modern.

Our closest living non-human relatives, the chimpanzees, also show a populationlevel bias towards right-handedness, although it is not nearly as marked as the roughly 9-to-1 ratio in humans ${ }^{27}$. Moreover, chimpanzees exhibit population-level asymmetries of some brain structures that are homologous to human brain structures that show asymmetry, including the planum temporale (a part of the temporal cerebral cortex that overlaps with Wernicke's classically defined language region $)^{28-30}$. The marked population-level bias in human handedness compared with that in chimpanzee handedness together with a modest association between left-handedness and atypical language dominance in humans (see below) have contributed to the hypothesis that language had its evolutionary origins in manual gesture ${ }^{31,32}$. However, language is only one of many cognitive processes that show a degree of lateralization of function in the human brain.

\section{To exclude, include or target?}

Within cognitive neuroscience, left-handers have a bad reputation: they are said to introduce unwanted noise in group-based studies. For example, language researchers commonly exclude left-handed participants in order to make their sample more homogeneous and to avoid including individuals with potentially reversed language lateralization (see below). Similarly, in neuroimaging studies, group analyses typically involve averaging neural measures (for example, blood-oxygen-level dependent (BOLD) responses from functional MRI (fMRI)) across participants. The reasoning is that if the sample includes participants with reversed lateralization, this will increase variance between subjects and hence reduce statistical sensitivity. Other fields of cognitive neuroscience may not exclude lefthanders a priori but may nevertheless do so a posteriori - for example, when a reversed laterality is observed in a participant.

In the field of human neurogenetics, a brief (non-systematic) search through literature published between January 2012 and May 2013 identified 13 studies in which left-handedness was applied as an exclusion criterion $^{33-43}$. Some of these studies focused on questions in cognitive neuroscience but included genetic variation as one variable of interest - an increasingly popular approach in human neuroscience research. We suggest that such studies have two potentially conflicting elements: they use left-handedness as an exclusion criterion to improve homogeneity in the study sample for groupbased analysis but at the same time aim to assess whether individual differences are due to genetic variation. The best way to assess the latter is to use a study sample that has a representative range of natural variability rather than a sample from which $10 \%$ of the population is excluded - particularly as left-handedness is a partly heritable trait (see below).

Our plea against the exclusion of lefthanded participants can be implemented in two, complementary ways. First, one could include left-handers in all studies in a proportion that corresponds to their population frequency (that is, roughly 10\%) - this is much like the balancing of participants' gender at 50/50, corresponding to the population level. We acknowledge that, for example, when testing 20 participants in an fMRI study, it will decrease the sensitivity of statistical group analysis to include 2 participants with potentially reversed lateralization of the function under study. However, we argue that this reflects a more general problem in group analyses of neuroimaging data - namely, the assumption that activation in response to a particular stimulus or during a particular task will occur in the same area in all participants. This assumption overlooks possible interesting differences in the localization of activity between participants (including between right-handed participants). To overcome this problem, it is important to develop and adopt methods of analysis at the level of the individual subject ${ }^{44}$ rather than the group level; this would then allow left-handers to be included in the study without negatively affecting statistical outcomes.

We suggest that the inclusion of lefthanded participants in neuroimaging experiments is most urgently required in studies that contribute to the establishment of large databases, including databases of structural and functional neuroimaging, and

\footnotetext{
Glossary

Ambidextrous

The ability to use both hands equally well. People of mixed handedness often do have hand preferences in individual tasks but no strong, overall bias across tasks.

Gene expression profiling

The measurement of mRNA expression of thousands of genes simultaneously using a quantitative technique.

\section{Imprinted}

An imprinted gene is a gene for which the copies that are inherited from father and mother are active to different degrees.

\section{Laterality index}

A quantitative index of left-right asymmetry. For handedness, this is typically based on questionnaires or tasks that assess degrees of hand preference or relative hand motor skill.

Lexical decision task

A task in which participants have to decide whether a
}

string of letters forms an existing word or not. The speed with which participants make their decision is used as a measure for semantic memory of a word or of the strength of association between words

\section{Pleiotropic}

Genetic effects that influence the development of multiple, distinct traits.

Praxis

The ability to perform purposeful movements. These movements can be learned gestures or a pantomime of tool use.

Situs inversus

A condition in which the position of visceral organs is reversed compared with that in the majority of the population

\section{Split-brain patients}

In split-brain patients, the corpus callosum (fibre bundles connecting the two hemispheres of the brain) is severely damaged or completely lesioned. This condition provides the possibility to study each hemisphere in isolation, providing insights into functional specialization of the hemispheres.

Tool-use pantomime task

A task in which participants are asked to mimic the hand movements that are related to tools presented on a screen. They execute the actions while holding an imaginary tool (for example, opening a bottle with a bottle opener).

Verb-generation task

A task in which participants are presented with a noun and have to generate verbs that go together with that noun (for example, for 'bread', responses could be 'eat' and 'slice').

Word-generation task

A task that is often used to study neural correlates of language production. In one form of this task, a letter is presented on the screen and the participant is asked to produce as many words starting with this letter as he or she can in a certain amount of time. 
cognitive and behavioural measures - especially if genetic information is also included in those databases. Below, we describe how studies that did include left-handers have provided new insights into the genetic basis of handedness and lateralization of the nervous system. We are aware of databases that contain anatomical and fMRI data in which the percentage of left-handed participants is lower than the proportion of left-handers in the population (for example, see the Cognomics website). A related recommendation for large-scale databases is to additionally record familial sinistrality, which is usually defined as having at least one left-handed parent or sibling. Studies have shown associations between familial sinistrality and both anatomical and functional brain asymmetries ${ }^{45-48}$. Familial sinistrality is attractive as a research tool for the study of brain asymmetries: there are many more individuals with familial sinistrality than there are left-handers.

Second, one could specifically target lefthanded participants, treating them as a distinct research sample and comparing them with a sample of right-handers. Below, we show that specifically recruiting left-handers as a target population is an informative strategy in cognitive neuroscience studies. Although this approach is not widely used, it enables researchers to investigate questions that cannot be addressed by studying right-handers alone. We review how targeted studies of left-handers can help to provide new insights into normal brain functioning, particularly in relation to embodied cognition, language processing and cerebral lateralization.

\section{Embodied cognition}

Embodied cognition posits that our body, including our motor behaviour, influences our cognition. One active area of research investigates how the sensorimotor cortex contributes to language understanding ${ }^{49}$. For example, when we read about someone running after a ball, does the motor cortex play a part in understanding this sentence? Generally speaking, research findings confirm that parts of the sensory or motor cortex show higher activation when people read vision- or action-related text compared with when they read language that is not related to action or to the senses. The interpretation of these findings is a topic of debate ${ }^{50-52}$, but this discussion is beyond the scope of this paper. Here, we focus on how findings from lefthanded participants have helped to increase our understanding of the nature of embodied semantic representations at the neural level.
Left-handers provide an excellent group of participants for testing how a person's motor expertise influences their representations of conceptual knowledge. Studies have shown activation of the left motor cortex when participants read about action verbs (for example, 'to throw' or 'to write') (53,54. $^{\text {. }}$ However, as all participants in those studies were right-handed, the left-lateralization could be due to motor expertise (participants prefer to perform hand actions with the right hand), to language lateralization or to their observation of others (of which the large majority were right-handers). Measuring brain activity in left-handers has made it possible to disentangle the influence of motor expertise from other factors ${ }^{55}$. For example, in an fMRI study, left- and right-handed participants performed a speeded lexical decision task on verbs related to hand actions ('manual'; to throw or to write) or non-handrelated actions ('non-manual'; to kneel or to laugh). Activation in the premotor cortex (Brodmann area 6) was differentially lateralized in the two groups of participants (FIG. 1). For right-handers, the difference in activation level in response to manual versus nonmanual action verbs was more pronounced in the left premotor cortex than in the right premotor cortex. The opposite was observed in left-handers ${ }^{55-57}$.

Another study investigated how hand preference influences the processing of tool sounds ${ }^{58}$. Left- and right-handers have an opposite hand preference for tool use (for example, hammering and sawing); according to the theory of embodied cognition, the neural correlates of the representation for tools should follow this preference. Indeed, when brain responses to tool sounds were contrasted with responses to animal sounds, there was a leftward lateralization in motor cortex activity in right-handers and a rightward lateralization of motor cortex activity in left-handers. Importantly, participants were not performing the actions implied by the tool sounds; they were simply listening to them.

Another example of the utility of investigating left-handed participants in studies of embodied cognition is provided by the finding that seeing single printed letters activates the motor system in a way that is compatible with writing preference ${ }^{59-61}$. Specifically, when left-handers passively viewed single letters (compared with pseudo-letters), the right ventral premotor cortex became activated, whereas the left ventral premotor cortex was activated in right-handed participants ${ }^{59-61}$. The authors suggested that reading simple letters involves an implicit mental simulation

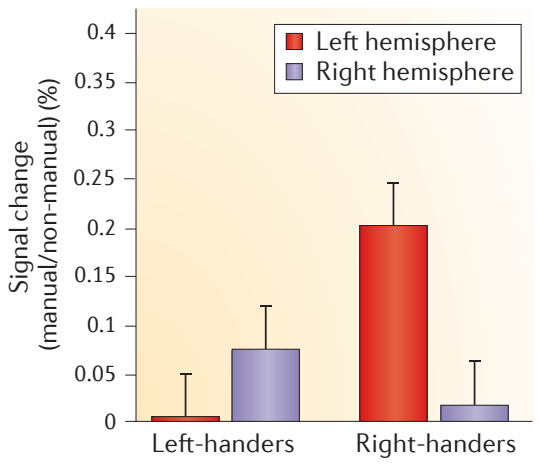

Figure 1 | Hemispheric activation differences in left- and right-handers during action verb reading. Participants were asked to read action verbs involving hand actions (for example, "to throw') or non-hand actions (for example, 'to laugh'). The graph shows the differences in activation in the premotor cortex (Brodmann area 6) between the two conditions. In right-handed participants, the difference in activation between hand-related and non-hand-related action conditions was most pronounced in the left hemisphere, which conforms to their hand preference (that is, right-handers preferentially use their right hand for performing manual actions, and motor control of the right hand is mainly governed by the left motor cortex). In left-handers, the pattern was reversed. Error bars represent SEM. There was a statistically significant threeway interaction effect between hemisphere (left versus right), group (left-handers versus righthanders) and action verb (manual versus nonmanual). Figure is reproduced, with permission, from REF. 55 ( ) (2010) SAGE Publications.

of actually writing those letters. Crucially, by comparing left- with right-handers, it was possible to show that the hypothesized motor simulation is sensitive to the personal writing preference of the reader.

Activation of left- and right-handers' motor cortex during action observation is the same as that observed during action execution of simple hand movements (opening and closing of the hands) ${ }^{62}$. This finding suggests that observation of simple hand movements occurs through an implicit simulation. Although this claim had been made previously on the basis of research in right-handed participants alone (for example, see REF. 63), the study that included lefthanders provided further evidence for it by showing that the motor simulation during simple action observation is specific to the observer's own motor repertoire ${ }^{62}$.

In another study of embodied cognition, left- and right-handers were asked to assign positive or negative characteristics to nonsense figures presented on the left or right side of a page. Left-handers attributed positive characteristics (for example, 
intelligence, happiness and honesty) more often to the figures presented on the left side of the page than to the figures on the right side. The response pattern was opposite for right-handers ${ }^{64,65}$. Again, this tells us how motor preference shapes our thinking, and comparing left- with right-handers can provide information that studying right-handers alone cannot. Together, these findings show that left-handers are useful subjects in studies of embodied cognition (for another example, see REF. 66).

\section{Cerebral lateralization}

Lateralization of language and motor functions. When, in the mid-1800s, Broca and Dax showed that speech production may be localized to a specific portion of the left hemisphere of the brain, scholars were quick to postulate a link between this finding and the lateralization of motor behaviour (with most people showing a right-hand preference). Researchers then argued that if speech is lateralized to the left hemisphere in righthanders, then it must be lateralized to the right hemisphere in left-handers. The idea that language is lateralized to the hemisphere opposite to the preferred hand was erroneously ${ }^{67}$ dubbed 'Broca's rule', and pioneering work in neuropsychology has robustly refuted it. Indeed, left-handed people suffered from problems with spoken language (aphasia) mostly after lesions or shot wounds to the left hemisphere rather than the right, in the same way as right-handers (for example, see REFS 68-70).

Modern research methods have largely confirmed the observation that language functions are left-lateralized in most people regardless of whether they are left- or right-handed. For example, one study used Doppler sonography to measure blood flow in left- and right-handed participants during performance of a word-generation task ${ }^{71}$. They reported that $96 \%$ of right-handed and $73 \%$ of left-handed participants showed stronger involvement of the left hemisphere than the right hemisphere. Thus, in the large majority of left-handers, language functions are left-lateralized, just as they are in righthanders. Nevertheless, left-handers show a greater diversity in language lateralization than right-handers: more left-handers show bilateral language function or have reversed lateralization $^{72-74}$.

In the majority of language-lateralization studies, 'language' is treated as a unitary cognitive function, and most investigations used speech-production tasks (such as a verb-generation task) to quantify levels of 'language'-related activation in frontal parts of the two hemispheres. However, besides the obvious distinction between production (speaking) and comprehension (listening), linguistic functions involve multiple subprocesses (such as word- or sentence-level comprehension) that recruit distinct neural systems with different localizations ${ }^{75-77}$. This raises the questions of whether there are varying degrees of lateralization for different linguistic functions and whether distinct sub-processes co-lateralize within an individual. Studies of right-handed split-brain patients have indicated that speech production is more strongly lateralized to the left hemisphere than reading; patients could read words presented in the left visual field (projecting to the right hemisphere) but were unable to name them ${ }^{78}$. Researchers have only recently started to address these intriguing questions further using healthy left-handed participants.

One important study compared the direction and degree of speech and reading lateralization in a cohort of 57 left-handers ${ }^{79}$. First, a lateralization index for speech production was calculated on the basis of activity in the left and right inferior frontal gyrus (that is, pars opercularis and triangularis) during a word-generation task. Thirty participants showed clear left-lateralization, twenty were clearly right-lateralized and seven showed bilateral activation in the regions of interest. The prior assessment of each subject's lateralization index for speech production ensured that the research sample included a considerable spread with respect to at least one sub-process of language.

The same 57 individuals then performed a lexical decision task in order to obtain lateralization indices for reading by comparing activation in the left and right ventral occipitotemporal cortex (sometimes referred to as the left and right 'visual word form area'). Most participants showed strong activation in the hemisphere that was their dominant hemisphere for speech production. However 31 participants showed bilateral patterns for word reading - that is, there was no clear activation dominance of the ventral occipitotemporal cortex when reading words - and strongly lateralized activation for speech production. Moreover, three participants processed speech and reading predominantly in opposite hemispheres ${ }^{79}$. These findings suggest that cerebral lateralization can differ for distinct sub-processes of a given cognitive function, at least in the case of the language system. Thus, the degree and/or direction of language lateralization depends on which aspect of the system is under investigation (see also REFS 80,81). Including only right-handers in a research sample will limit both the degree and direction of lateralization indices and thereby mask possible variability in lateralization patterns.

Another study investigated the relationship between lateralization of different cognitive systems - namely, those for speech production and spatial attention - within the same individuals ${ }^{82}$. The study involved left-handed participants with previously established differences in lateralization during speech production. Participants performed a word-generation task and the so-called 'landmark' task, in which they had to decide whether a horizontal line was bisected exactly in the middle by another line - this task was used to map regions involved in visuospatial attention. A comparison of lateralization in the inferior frontal cortex (which is involved in speech production) with that in parietal areas (involved in spatial attention), which tend to be right-lateralized, revealed that lateralization in one domain was associated with lateralization of the other function in the other hemisphere (FIG. 2). Fifteen out of sixteen left-handers who were leftlateralized for speech were right-lateralized for spatial attention, whereas all thirteen left-handers who were right-lateralized for speech showed left-hemispheric dominance for spatial attention.

There is an apparent contradiction between this complementary lateralization and the independence of lateralization of speech and spatial attention suggested by findings from other studies ${ }^{83-85}$. An important difference between the two sets of studies is that in the recent studies discussed above, left-handers were pre-screened and subsequently selected on the basis of their speech lateralization to ensure sufficient variability in the lateralization indices of the study sample ${ }^{86}$. As the variability of speech lateralization in left-handers is much greater than that in right-handers, left-handers are an obvious target population for these studies. However, pre-selection of a study sample raises the question of whether the findings will generalize to the whole population, including both left- and right-handers.

It was long assumed that lateralization of tool-use actions is related to hand preference, because right-handed patients with apraxia (who are unable to perform learned gestures) all seemed to have left-hemispheric lesions. However, subsequent publication of cases of right-handed patients with righthemispheric lesions ${ }^{87}$ and left-handers with left-hemispheric lesions ${ }^{88}$ contradicted this assumption. Recent studies in left- and 
a Typical lateralization Left hemisphere

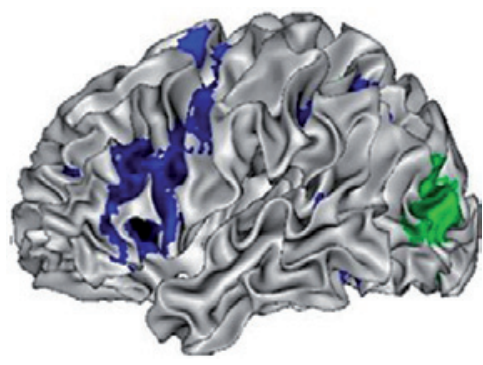

b Atypical lateralization Left hemisphere

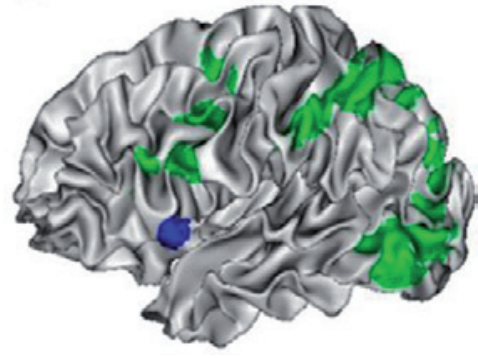

Right hemisphere

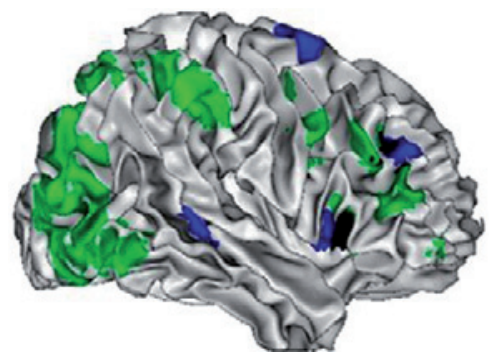

Right hemisphere

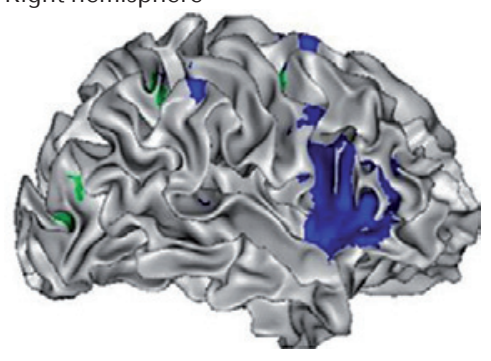

Language production

Visuospatial attention

Figure 2 | Language and visuospatial activations in left-handers with typical and atypical language lateralization. Activation in the language network during a word-generation task is shown in blue. Left-handed individuals with typical language lateralization (panel a) show more activation in left-hemispheric language regions than in right-hemispheric regions, whereas the reverse is true for left-handed individuals with atypical, right-lateralized language function (panel b). A set of regions activated during a visuospatial attention task (shown in green) was also differentially lateralized in these two groups. In left-handed individuals in whom language is left-lateralized (typical), activation was most pronounced in the right hemisphere (panel a), whereas in left-handed individuals in whom language was right-lateralized (atypical), activation was most pronounced in the left hemisphere (panel $\mathbf{b}$ ). This study was conducted in left-handers because of the greater variability in language lateralization in left-handers. By actively looking for atypically lateralized individuals within the left-handed population, the issue of co-lateralization of linguistic and visuospatial functions could be addressed. Figure is reproduced from REF. 82.

right-handed healthy subjects reported that the lateralization of complex movements may be linked to language dominance rather than handedness ${ }^{86,89}$. The degree and direction of lateralization of praxis (as measured in a tool-use pantomime task) correlated with the degree and direction of lateralization of speech production (as measured in a wordgeneration task) ${ }^{89}$. This co-lateralization occurred in all individuals with typical left-lateralized dominance for speech (in this study these individuals consisted of one right-hander, one ambidextrous person and eight left-handers) and in an atypical sample of individuals with right-lateralization for speech who were matched for handedness. In other words, although there was no effect of handedness in this study, the inclusion of left-handers with different directions and degrees of speech lateralization meant that the study sample had sufficient variability to test whether the lateralization of language and the lateralization of praxis are linked.
The most obvious neural system in which to compare left- and right-handers is the motor system. After all, by definition left- and right-handers differ in their motor repertoire and preferences, and this should be reflected in some way at the neural level. Left- and right-handers show similar activation patterns in the cortical motor network during the execution of simple hand actions, with left-handers showing a less strong and more variable lateralization ${ }^{90-93}$. One study showed that there was a difference in lateralization in ipsilateral premotor cortex activation between left- and right-handers ${ }^{94}$. Left dorsal premotor cortex activation during movement with the left hand was stronger than right dorsal premotor cortex activation during movement of the right hand - this was the case for both left- and right-handers, but the difference was smaller in left-handed participants ${ }^{94}$. A converging but tentative picture emerging from these findings is that left- and right-handers do not differ considerably in brain lateralization during simple, unimanual movements but that differences emerge for more complex and/or bimanual movement patterns.

Taken together, these studies show that the inclusion of left-handers in study samples can open exciting and new avenues for studying the relationships between apparently distinct neural systems; specifically, it can reveal which systems co-lateralize and which do not, and thereby shed light on functional and/or evolutionary links between them.

Lateralization in the visual system. An often-cited functional lateralization in the brain concerns the processing of faces, which activates ventral occipital areas in the right hemisphere ${ }^{95}$. This functional asymmetry is heritable ${ }^{96}$, is stable within individuals ${ }^{97}$ and is also present in macaque monkeys ${ }^{98}$. As such, it can be thought of as a fundamental lateralization of the brain. Intriguingly, left-handers show a different lateralization of brain activation during face processing ${ }^{85,99}$. In one study, fMRI was used to localize areas involved in face and body perception in a sample of participants, half of whom were left-handers ${ }^{99}$. In the right-handers, there was much larger activation in the right fusiform face area and extrastriate body area than in their left-hemisphere counterparts in response to pictures of faces and bodies, respectively. By contrast, in the left-handers, activation in these areas was similar in the two hemispheres (FIG. 3). A related study using a larger sample size showed that rather than having a reversed lateralization, left-handers had a smaller rightward lateralization compared with righthanders. One tentative explanation for the difference between the two studies is that in the latter study ${ }^{85}$, the presented pictures of faces expressed emotions, a suggestion that requires further research.

The difference in lateralization of face- and body-related visual areas between left-handers and right-handers is intriguing: it means that around $10 \%$ of the general population (that is, left-handers) does not exhibit an aspect of lateralization that is assumed to be a basic feature of visual processing in humans and other primates. It also indicates that, relatively early in the course of visual processing, lateralization can be present or absent without obvious behavioural consequences.

\section{Genetics of brain lateralization}

As noted above, the strong population-level bias towards right-handedness is evident throughout human history, across continents and ethnicities, and across the human lifespan from early in gestation. This motor 


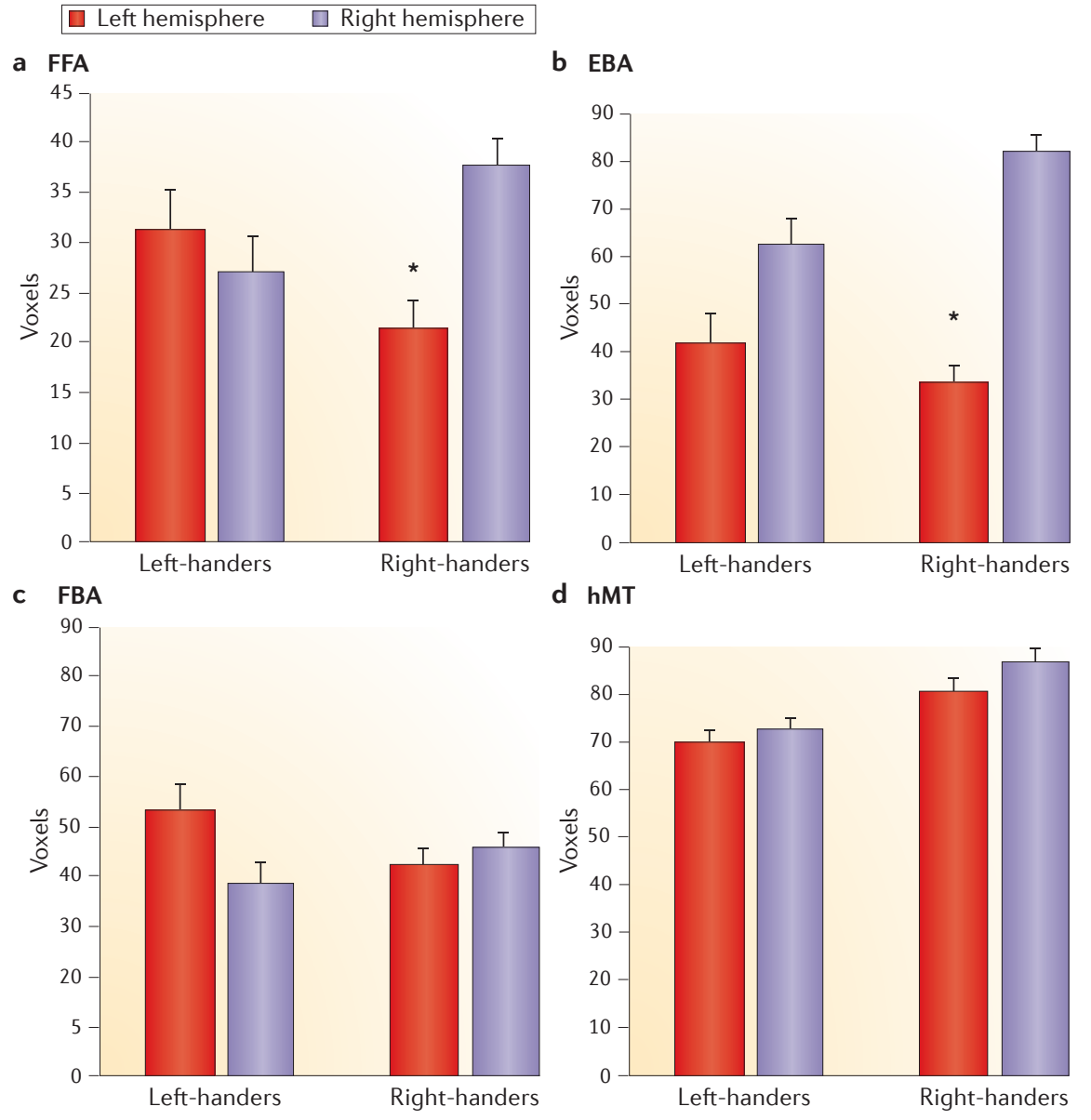

Figure 3 | Left- and right-handers show differences in lateralization during face perception. Parts of the extrastriate cortex are selectively sensitive to the perception of faces or bodies, and these areas are sometimes dubbed the fusiform face area (FFA), and the extrastriate body area (EBA) and fusiform body area (FBA), respectively. The FFA in particular is thought to be right-lateralized, and the figure shows that this may in fact not be the case for left-handers. Left- and righthanded participants were asked to view pictures of faces, bodies and chairs (control stimuli), and the extent of activation was quantified. The graphs show the extent of activation (in number of voxels on the $y$ axis) in four extrastriate visual areas when participants viewed faces or bodies compared with the extent of activation during the viewing of chairs. a | The typical right-lateralization of the FFA in right-handers is absent in left-handers. b| Right-handers show a similar rightlateralization in the EBA, and left-handers again show no statistically significant lateralization in this area. $\mathbf{c}$ |There is no statistically significant lateralization in the FBA in either left-handers or right-handers. $\mathbf{d}$ | The human motion area MT (hMT), which is a visual area that is sensitive to motion, also did not show any lateralization effect of handedness. These findings indicate that right-lateralization does not occur in all functional areas in the visual system but is specific for the FFA and EBA in right-handers. Statistically significant differences between activity in the left versus right hemisphere are indicated by an asterisk. Figure is reproduced, with permission, from REF. 99 (c) (2010) Oxford University Press.

asymmetry is therefore likely to be related to left-right asymmetrical development of the nervous system that is initiated early in human embryogenesis. The genetic basis of asymmetrical human brain development is unknown, although some potentially relevant genes have been identified through gene expression profiling from the left and right cerebral cortex in post-mortem human foetuses $^{100}$. Here, we illustrate how the inclusion of left-handers in behavioural and neurogenetics studies has provided further insights into the genetic architecture of this trait. For a more detailed review of genetics studies of handedness, see REF. 101.

By comparing thousands of identical and non-identical twins, left-handedness has been shown to have a highly significant heritability of approximately $25 \%{ }^{102}$. Genome-wide association studies (GWASs) carried out using thousands of subjects have not identified any DNA variant that, by itself, makes a substantive contribution to lefthandedness ${ }^{103,104}$. The heritability studies and GWASs involved meta-analyses of datasets from primary studies that themselves did not focus on handedness; rather, in these primary studies, handedness was assessed as a secondary consideration - and fortunately not as an exclusion criterion. The GWASs indicate that any genetic effects on handedness that arise from common polymorphisms in the population are likely to be extremely small. However, there may be a heterogeneous set of genetic variants that individually have substantial effects on handedness but that are each relatively rare in the population $^{104,105}$. Such variants would therefore not have large effects at the population level but may nonetheless be important contributors to handedness when they are present ${ }^{104}$.

One study ${ }^{106}$ reported the first candidate gene that may contribute to human handedness: leucine-rich repeat transmembrane neuronal 1 (LRRTM1). Variants of this gene were associated with a quantitative measure of relative hand skill (a performance-based measure), which was assessed using a task in which pegs are moved from one side of a board to another ${ }^{7}$. The study used a dataset of families in which at least one of the siblings had dyslexia and in which relative hand skill had been assessed. Left-handers had not been excluded, so that the dataset contained the full range of relative hand skill. The associations between LRRTM1 variants and handedness seemed to be specific for the gene variants that had been inherited from the father's side (that is, genes of paternal origin), suggesting that the gene might be paternally imprinted. LRRTM1 regulates the differentiation of excitatory synapses ${ }^{107-112}$ and binds to neurexin proteins across the synaptic cleft ${ }^{107-112}$. Genetic deletion mapping strongly implicates neurexins in a range of major neuropsychiatric disorders, including autism and schizophrenia, both of which are associated with increased rates of lefthandedness and alterations of structural and functional brain asymmetry ${ }^{113-125}$ (although not in all clinical populations ${ }^{126}$ ). However, it is not yet known how the LRRTM1-neurexin pathway might be involved in asymmetrical development or functioning of the brain.

In follow-up genome-wide studies of the same dataset of families in which at least one of the siblings had dyslexia, researchers uncovered a second candidate gene for handedness, PCSK6 (proprotein convertase subtilisin/kexin type 6$)^{127}$. This gene was also associated with relative hand skill but did not 
seem to be subject to imprinting and may be linked to the degree rather than direction of handedness ${ }^{128}$. PCSK6 encodes a protease enzyme that cleaves Nodal, a protein that has a role in the regulation of the left-right visceral axis (of the heart, lungs, and so on) in early embryogenesis ${ }^{127}$. GWAS analysis of this same dataset, in combination with data from other cohorts, has provided evidence that additional genes involved in left-right visceral patterning are associated with relative hand skill ${ }^{129}$. These studies provide a hint that human handedness and visceral asymmetries may be genetically connected, at least to some extent. Previously, it was thought that visceral asymmetry and right-handedness were developmentally disconnected, because people with situs inversus due to rare mutations that cause randomized asymmetry of the viscera have proportions of right-handedness ${ }^{130}$ and lateralized auditory dominance ${ }^{131}$ similar to that of the normal population.

Clearly, the need for further validation of genetic associations with handedness, which have so far typically arisen from opportunistic analysis in datasets that were collected for quite other reasons, underscores the need not to impose an unnecessary exclusion criterion of left-handedness in the next generation of behavioural, psychiatric and brain imaging genetics studies.

Given that left-handedness has a heritability of $\sim 25 \%$, a large non-genetically determined component must also be involved ${ }^{102}$. As is the case for genetic factors, any relevant environmental influences are most probably heterogeneous in nature. One possible contributor is low birth weight, although this effect is apparently small ${ }^{102}$. It is also worth noting that an association between handedness and birth weight is not necessarily due to purely environmental effects, as pleiotropic genetic factors may be involved. Another possibility, much discussed in the literature, is that maternal levels of androgens influence in utero the development of handedness and brain asymmetry in human embryos and foetuses ${ }^{132,133}$. Finally, as motor and brain asymmetries appear early in human development, the relatively low heritability of left-handedness may reflect developmental processes that have a substantial random component and occur on a small scale in the embryo during early differentiation. In twin studies, such random effects that occur early in development are considered to be an 'environmental' component of trait susceptibility. One of our key reasons for advocating genetics studies that include or specifically target left-handers is that such studies will lead to a greater understanding of asymmetrical developmental processes in the human nervous system - processes that are currently a fundamental mystery in the developmental biology of our species.

\section{Conclusions and future directions}

We argue that left-handers should not be systematically excluded from research programmes and study collections in cognitive neuroscience and neurogenetics. Their inclusion will have broad and substantial benefits across multiple fields of enquiry.

We have illustrated how left-handers can be used to develop and test hypotheses in cognitive neuroscience. For example, lefthanders have proven to be valuable in studies testing the role of motor expertise on cognition (embodied cognition). Importantly, left-handed participants tend to have more atypically lateralized brain functions, which makes it possible to study not only basic lateralization of brain function (for example, of face perception) but also the presence or absence of co-lateralization of different cognitive functions. Such studies should be greatly aided in the future by the availability of large-scale databases that contain fMRI data from many subjects. Crucially, left-handed participants should be included in such databases, as they will infuse the necessary variation needed to test co-lateralization, both within neural systems and between them.

We have described how left-handers can also form an important element of genetics research into the molecular mechanisms of asymmetrical brain development; we propose that molecular studies of left-handedness hold great potential for identifying genes that are important for cerebral lateralization. It will be particularly promising to identify large extended families with an increased rate of left-handedness across various branches of the family tree, because such families may reveal genetic sub-forms of this trait and this may lead to the identification of crucial molecular networks and pathways in cerebral lateralization. As we enter the era of personal genome sequencing, we have an unprecedented opportunity to decode the genetic underpinnings of traits that are genetically heterogeneous at the population level.

Genetic association studies of human brain structure and function are just now starting to be performed in samples of thousands of subjects (for examples, see REFS 134,135). Many of the imaging-based genetics studies that were published previously are likely to have been underpowered to detect the realistically tiny effects of common genetic variations, and a lot of statistical noise and publication bias is likely to have affected the literature ${ }^{136}$. Again, adequately powered imaging-based genetics studies are only achievable through the combined analysis of smaller contributing datasets, which are collected with their own priorities and focuses. However, if large-scale genetics studies of structure and function are to capture normal human variation adequately, it is important that left-handedness should not be applied as an exclusion criterion in the individual, smaller studies.

Roel M. Willems, Simon E. Fisher and Clyde Francks are at the Donders Institute for Brain, Cognition and Behaviour, Radboud University Nijmegen, 6525 EN Nijmegen, The Netherlands, and the Max Planck Institute for Psycholinguistics, 6525 XD Nijmegen, The Netherlands.

Lise Van der Haegen is at the Department of Experimental Psychology, Ghent University, Ghent, 9000, Belgium

Correspondence to R.M.W. and C.F. e-mails: roel.willems@donders.ru.nl; clyde.francks@mpi.nl doi:10.1038/nrn3679 Published online 12 February 2014

1. McManus, I. C. Right Hand, Left Hand (Phoenix, 2002)

2. Smits, R. The Puzzle of Left-Handedness (Reaktion Books, 2011)

3. Blau, A. Don't let your child be a lefty! Tri-City Herald (Washington) 38 (1961).

4. Oldfield, R. C. The assessment and analysis of handedness: the Edinburgh inventory. Neuropsychologia 9, 97-113 (1971).

5. Büsch, D., Hagemann, N. \& Bender, N. The dimensionality of the Edinburgh Handedness Inventory: an analysis with models of the item response theory. Laterality 15, 610-628 (2010).

6. Dragovic, M. Towards an improved measure of the Edinburgh Handedness Inventory: a one-factor congeneric measurement model using confirmatory factor analysis. Laterality 9, 411-419 (2004).

7. Annett, M. Left, Right, Hand and Brain: The Right Shift Theory (Laurence Erlbaum, 1985).

8. Annett, M. Patterns of hand preference for pairs of actions and the classification of handedness. $\mathrm{Br}$ J. Psychol. 100, 491-500 (2009).

9. Nicholls, M. E. R., Chapman, H. L., Loetscher, T. \& Grimshaw, G. M. The relationship between hand preference, hand performance, and general cognitive ability. J. Int. Neuropsychol. Soc. 16, 585-592 (2010).

10. Triggs, W. J., Calvanio, R., Levine, M., Heaton, R. K. \& Heilman, K. M. Predicting hand preference with performance on motor tasks. Cortex 36, 679-689 (2000).

11. Björk, T., Brus, O., Osika, W. \& Montgomery, S. Laterality, hand control and scholastic performance: a British birth cohort study. BMJ Open 2, e000314 (2012).

12. Badzakova-Trajkov, G., Häberling, I. S. ¿ Corballis, M. C. Magical ideation, creativity, handedness, and cerebral asymmetries: a combined behavioural and fMRI study. Neuropsychologia 49, 2896-2903 (2011).

13. Somers, M., Sommer, I. E., Boks, M. P. \& Kahn, R. S. Hand-preference and population schizotypy: a metaanalysis. Schizophr. Res. 108, 25-32 (2009).

14. Eckert, M. A. et al. Manual and automated measures of superior temporal gyrus asymmetry: concordant structural predictors of verbal ability in children. Neuroimage 41, 813-822 (2008).

15. Groen, M. A., Whitehouse, A. J. O., Badcock, N. A. \& Bishop, D. V. M. Does cerebral lateralization develop? A study using functional transcranial Doppler ultrasound assessing lateralization for language production and visuospatial memory. Brain Behav. 2, 256-269 (2012) 
16. Bryden, M. P., Roy, E. A., McManus, I. C. \& Bulman Fleming, M. B. On the genetics and measurement of human handedness. Laterality 2, 317-336 (1997).

17. Reiss, M., Tymnik, G., Kögler, P., Kögler, W. \& Reiss, G. Laterality of hand, foot, eye, and ear in twins. Laterality 4, 287-297 (1999).

18. Elias, L. J. \& Bryden, M. P. Footedness is a better predictor of language lateralisation than handedness. Laterality 3, 41-51 (1998).

19. Mcmanus, I. C. in Language Lateralization and Psychosis (eds Sommer, I. E. C. \& Kahn, R. S.) 37-57 (Cambridge Univ. Press, 2009).

20. Faurie, C. \& Raymond, M. Handedness frequency over more than ten thousand years. Proc. R. Soc. Lond. B 271, S43-S45 (2004).

21. Perelle, I. B. \& Ehrman, L. An international study of human handedness: the data. Behav. Genet. 24, 217-227 (1994)

22. Hepper, P. G., McCartney, G. R. \& Shannon, E. A Lateralised behaviour in first trimester human foetuses. Neuropsychologia 36, 531-534 (1998).

23. Hepper, P. G. The developmental origins of laterality: fetal handedness. Dev. Psychobiol. 55, 588-595 (2013).

24. Hugdahl, K. \& Davidson, R. J. The Asymmetrical Brain (MIT Press, 2004).

25. Hering-Hanit, R., Achiron, R., Lipitz, S. \& Achiron, A Asymmetry of fetal cerebral hemispheres: in utero ultrasound study. Arch. Dis. Child. Fetal Neonatal Ed. 85, F194-F196 (2001).

26. Rogers, L. J. \& Andrew, R. (eds) Comparative Vertebrate Lateralization (Cambridge Univ. Press, 2002).

27. Hopkins, W. D. et al. Hand preferences for coordinated bimanual actions in 777 great apes: implications for the evolution of handedness in hominins. J. Hum. Evol 60, 605-611 (2011).

28. Gannon, P. J., Holloway, R. L., Broadfield, D. C. \& Braun, A. R. Asymmetry of chimpanzee planum temporale: humanlike pattern of Wernicke's brain language area homolog. Science 279, 220-222 (1998).

29. Hopkins, W. D. et al. Gray matter asymmetries in chimpanzees as revealed by voxel-based morphometry. Neuroimage 42, 491-497 (2008).

30. Lyn, H. et al. Planum temporale grey matter asymmetries in chimpanzees (Pan troglodytes), vervet (Chlorocebus aethiops sabaeus), rhesus (Macaca mulatta) and bonnet (Macaca radiata) monkeys. Neuropsychologia 49, 2004-2012 (2011).

31. Corballis, M. C. From mouth to hand: gesture, speech, and the evolution of right-handedness. Behav. Brain Sci. 26, 199-208 (2003)

32. Corballis, M. C., Badzakova-Trajkov, G. \& Häberling, I. S Right hand, left brain: genetic and evolutionary bases of cerebral asymmetries for language and manual action. Cogn. Sci. 3, 1-17 (2012)

33. Pinel, P. et al. Genetic variants of FOXP2 and KIAAO319/TTRAP/THEM2 locus are associated with altered brain activation in distinct language-related regions. J. Neurosci. 32, 817-825 (2012).

34. Kos, M. et al. CNTNAP2 and language processing in healthy individuals as measured with ERPs. PLOS ONE 7, e46995 (2012).

35. Kim, B. et al. The effects of the catechol$O$-methyltransferase val 158 met polymorphism on white matter connectivity in patients with panic disorder. J. Affect. Disord. 147, 64-71 (2013)

36. Rose, E. J. et al. The effect of the neurogranin schizophrenia risk variant rs 12807809 on brain structure and function. Twin Res. Hum. Genet. 15 296-303 (2012).

37. Hibar, D. P. et al. Alzheimer's disease risk gene, GAB2, is associated with regional brain volume differences in 755 young healthy twins. Twin Res. Hum. Genet. 15 286-295 (2012)

38. Chen, J. et al. A combined study of genetic association and brain imaging on the $D A O A$ gene in schizophrenia. Am. J. Med. Genet. B Neuropsychiatr. Genet. 162 191-200 (2013)

39. Sprooten, E. et al. An investigation of a genomewide supported psychosis variant in ZNF804A and white matter integrity in the human brain. Magn. Reson. Imag. 30, 1373-1380 (2012)

40. Yang, X et al. Impact of brain-derived neurotrophic factor Val66Met polymorphism on cortical thickness and voxel-based morphometry in healthy Chinese young adults. PLoS ONE 7, e37777 (2012).

41. Li, Y. et al. Less efficient information transfer in Cysallele carriers of DISC 1: a brain network study based on diffusion MRI. Cereb. Cortex 23, 1715-1723 (2013).
42. Paulus, F. M. et al. Association of rs 1006737 in CACNA $1 C$ with alterations in prefrontal activation and fronto-hippocampal connectivity. Hum. Brain Mapp. http://dx.doi.org/10.1002/hbm.22244 (2013).

43. van der Heijden, C. D. C. C. et al. Genetic variation in ataxia gene $A T X N 7$ influences cerebellar grey matter volume in healthy adults. Cerebellum 12, 390-395 (2013).

44. Nieto-Castañón, A. \& Fedorenko, E. Subject-specific functional localizers increase sensitivity and functional resolution of multi-subject analyses. Neuroimage 63 1646-1669 (2012)

45. Hancock, R. \& Bever, T. G. Genetic factors and normal variation in the organization of language. Biolinguistics 7, 075-095 (2013)

46. Townsend, D. J., Carrithers, C. \& Bever, T. G. Familial handedness and access to words, meaning, and syntax during sentence comprehension. Brain Lang. 78, 308-331 (2001).

47. Tzourio-Mazoyer, N. et al. Left hemisphere lateralization for language in right-handers is controlled in part by familial sinistrality, manual preference strength, and head size. J. Neurosci. 30 , 13314-13318 (2010)

48. Tzourio-Mazoyer, N. et al. Effect of familial sinistrality on planum temporale surface and brain tissue asymmetries. Cereb. Cortex 20, 1476-1485 (2010).

49. Willems, R. M. \& Casasanto, D. Flexibility in embodied language understanding. Front. Psychol. 2 , 116 (2011).

50. Mahon, B. Z. \& Caramazza, A. A critical look at the embodied cognition hypothesis and a new proposal for grounding conceptual content. J. Physiol. Paris 102 , 59-70 (2008).

51. Willems, R. M \& Francken, J. C. Embodied cognition: taking the next step. Front. Cogn. Sci. 3, 582 (2012)

52. Willems, R. M. \& Hagoort, P. Neural evidence for the interplay between language, gesture, and action: a review. Brain Lang. 101, 278-289 (2007).

53. Aziz-Zadeh, L., Wilson, S. M., Rizzolatti, G. \& lacoboni, M. Congruent embodied representations for visually presented actions and linguistic phrases describing actions. Curr. Biol. 16, 1818-1823 (2006).

54. Hauk, O., Johnsrude, I. \& Pulvermuller, F. Somatotopic representation of action words in human motor and premotor cortex. Neuron 41, 301-307 (2004).

55. Willems, R. M., Hagoort, P. \& Casasanto, D. Bodyspecific representations of action verbs: neural evidence from right- and left-handers. Psychol. Sci. 21 67-74 (2010)

56. Willems, R. M., Toni, I., Hagoort, P. \& Casasanto, D. Body-specific motor imagery of hand actions: neural evidence from right- and left-handers. Front. Hum. Neurosci. 3, 39 (2009).

57. Hauk, O. \& Pulvermüller, F. The lateralization of motor cortex activation to action-words. Front. Hum. Neurosci. 5, 149 (2011)

58. Lewis, J. W., Phinney, R. E., Brefczynski-Lewis, J. A. \& DeYoe, E. A. Lefties get it 'right' when hearing tool sounds. J. Cogn. Neurosci. 18, 1314-1330 (2006).

59. Longcamp, M., Anton, J. L., Roth, M. \& Velay, J. L. Visual presentation of single letters activates a premotor area involved in writing. Neuroimage 19 1492-1500 (2003)

60. Longcamp, M., Anton, J. L., Roth, M. \& Velay, J. L. Premotor activations in response to visually presented single letters depend on the hand used to write: a study on left-handers. Neuropsychologia 43, 1801-1809 (2005).

61. Longcamp, M., Tanskanen, T. \& Hari, R. The imprint of action: motor cortex involvement in visual perception of handwritten letters. Neuroimage 33, 681-688 (2006)

62. Willems, R. M. \& Hagoort, P. Hand preference influences neural correlates of action observation. Brain Res. 1269, 90-104 (2009).

63. Hari, R. et al. Activation of human primary motor cortex during action observation: a neuromagnetic study. Proc. Natl Acad. Sci. USA 95, 15061-15065 (1998).

64. Casasanto, D. Embodiment of abstract concepts: good and bad in right- and left-handers. J. Exp. Psychol. Gen. 138, 351-367 (2009).

65. Casasanto, D. Different bodies, different minds the body specificity of language and thought. Curr. Dir. Psychol. Sci. 20, 378-383 (2011)

66. De Nooijer, J. A., van Gog, T., Paas, F. \& Zwaan, R. A. When left is not right: handedness effects on learning object-manipulation words using pictures with left- or right-handed first-person perspectives. Psychol. Sci. 24, 2515-2521 (2013).
67. Eling, P. Broca on the relation between handedness and cerebral speech dominance. Brain Lang. 22, 158-159 (1984)

68. Ettlinger, G., Jackson, C. V. \& Zangwill, O. L. Cerebral dominance in sinistrals. Brain 79, 569-588 (1956).

69. Goodglass, H. \& Quadfasel, F. A. Language laterality in left-handed aphasics. Brain 77, 521-548 (1954).

70. Hécaen, H., De Agostini, M. \& Monzon-Montes, A. Cerebral organization in left-handers. Brain Lang. 12, 261-284 (1981).

71. Knecht, S. et al. Handedness and hemispheric language dominance in healthy humans. Brain 123 2512-2518 (2000)

72. Szaflarski, J. P. et al. Language lateralization in lefthanded and ambidextrous people: fMRI data. Neurology 59, 238-244 (2002)

73. Steinmetz, H., Volkmann, J., Jäncke, L. \& Freund, H. J. Anatomical left-right asymmetry of language-related temporal cortex is different in left- and right-handers. Ann. Neurol. 29, 315-319 (1991).

74. Sommer, I. E. C., Ramsey, N. F., Mandl, R. C. W. \& Kahn, R. S. Language lateralization in monozygotic twin pairs concordant and discordant for handedness. Brain 125, 2710-2718 (2002).

75. Bookheimer, S. Functional MRI of language: new approaches to understanding the cortical organization of semantic processing. Annu. Rev. Neurosci. 25 151-188 (2002)

76. Price, C. J. The anatomy of language: a review of 100 fMRI studies published in 2009. Ann. NY Acad. Sci. $1191,62-88$ (2010).

77. Hagoort, P., Baggio, G. \& Willems, R. M. in The Cognitive Neurosciences 4th edn (ed. Gazzaniga, M. S.) 819-836 (MIT Press, 2009).

78. Sperry, R. Some effects of disconnecting the cerebral hemispheres. Science 217, 1223-1226 (1982).

79. Van der Haegen, L., Cai, Q. \& Brysbaert, M. Colateralization of Broca's area and the visual word form area in left-handers: fMRI evidence. Brain Lang. 122, 171-178 (2012).

80. Seghier, M. L., Kherif, F., Josse, G. \& Price, C. J. Regional and hemispheric determinants of language laterality: implications for preoperative fMRI. Hum. Brain Mapp. 32, 1602-1614 (2011)

81. Tzourio-Mazoyer, N., Josse, G., Crivello, F. \& Mazoyer, B. Interindividual variability in the hemispheric organization for speech. Neuroimage 21 422-435 (2004)

82. Cai, Q., Van der Haegen, L. \& Brysbaert, M. Complementary hemispheric specialization for language production and visuospatial attention. Proc Natl Acad. Sci. USA 110, E322-E330 (2013).

83. Whitehouse, A. J. O. \& Bishop, D. V. M. Hemispheric division of function is the result of independent probabilistic biases. Neuropsychologia 47 1938-1943 (2009).

84. Bryden, M. P., Hécaen, H. ¿ DeAgostini, M. Patterns of cerebral organization. Brain Lang. 20, 249-262 (1983).

85. Badzakova-Trajkov, G., Häberling, I. S., Roberts, R. P. \& Corballis, M. C. Cerebral asymmetries: complementary and independent processes. PLoS ONE 5, e9682 (2010).

86. Króliczak, G., Piper, B. J. \& Frey, S. H. Atypical lateralization of language predicts cerebral asymmetries in parietal gesture representations. Neuropsychologia 49, 1698-1702 (2011).

87. Raymer, A. M. et al. Crossed apraxia: implications for handedness. Cortex 35, 183-199 (1999).

88. Goldenberg, G. Apraxia - the cognitive side of motor control. Cortex http://dx.doi.org/10.1016/j. cortex.2013.07.016 (2013).

89. Vingerhoets, G. et al. Praxis and language are linked: evidence from co-lateralization in individuals with atypical language dominance. Cortex 49, 172-183 (2013).

90. Van den Berg, F. E., Swinnen, S. P. \& Wenderoth, N Involvement of the primary motor cortex in controlling movements executed with the ipsilateral hand differs between left- and right-handers. J. Cogn. Neurosci. 23, 3456-3469 (2011)

91. Kloppel, S. et al. The effect of handedness on cortical motor activation during simple bilateral movements. Neuroimage 34, 274-280 (2007)

92. Solodkin, A., Hlustik, P., Noll, D. C. \& Small, S. L. Lateralization of motor circuits and handedness during finger movements. Eur. J. Neurol. 8, 425-434 (2001)

93. Dassonville, P., Zhu, X. H., Uurbil, K., Kim, S. G. \& Ashe, J. Functional activation in motor cortex reflects the direction and the degree of handedness. Proc. Natl Acad. Sci. USA 94, 14015-14018 (1997). 
94. Verstynen, T., Diedrichsen, J., Albert, N., Aparicio, P. \& Ivry, R. B. Ipsilateral motor cortex activity during unimanual hand movements relates to task complexity. J. Neurophysiol. 93, 1209-1222 (2005).

95. Corballis, M. C. The Lopsided Ape: Evolution of the Generative Mind (Oxford Univ. Press, 1991).

96. Polk, T. A., Park, J., Smith, M. R. \& Park, D. C. Nature versus nurture in ventral visual cortex: a functional magnetic resonance imaging study of twins. J. Neurosci. 27, 13921-13925 (2007).

97. Yovel, G., Tambini, A. \& Brandman, T. The asymmetry of the fusiform face area is a stable individual characteristic that underlies the left-visual-field superiority for faces. Neuropsychologia $\mathbf{4 6}$, 3061-3068 (2008).

98. Hamilton, C. R. \& Vermeire, B. A. Complementary hemispheric specialization in monkeys. Science $\mathbf{2 4 2}$, 1691-1694 (1988).

99. Willems, R. M., Peelen, M. V. \& Hagoort, P. Cerebral lateralization of face-selective and body-selective visual areas depends on handedness. Cereb. Cortex 20, 1719-1725 (2010).

100. Sun, T. et al. Early asymmetry of gene transcription in embryonic human left and right cerebral cortex. Science 308, 1794-1798 (2005).

101. Ocklenburg, S., Beste, C. \& Güntürkün, O Handedness: a neurogenetic shift of perspective. Neurosci. Biobehav. Rev. 37, 2788-2793 (2013).

102. Medland, S. E. et al. Genetic influences on handedness: data from 25,732 Australian and Dutch twin families. Neuropsychologia 47, 330-337 (2009).

103. Medland, S. E. et al. Meta-analysis of GWAS for handedness: results from the ENGAGE consortium. Am. Soc. Hum. Genet. Abstr. [online], http://www.ashg. org/2009meeting/abstracts/fulltext/f21141.htm (2009).

104. Armour, J. A. L., Davison, A. \& McManus, I. C. Genome-wide association study of handedness excludes simple genetic models. Heredity http://dx. doi.org/10.1038/hdy.2013.93 (2013).

105. Singleton, A. B., Hardy, J., Traynor, B. J. \& Houlden, H. Towards a complete resolution of the genetic architecture of disease. Trends Genet. 26, 438-442 (2010).

106. Francks, C. et al. LRRTM 1 on chromosome $2 \mathrm{p} 12$ is a maternally suppressed gene that is associated paternally with handedness and schizophrenia. Mol Psychiatry 12, 1129-1139 (2007).

107. Francks, C. Leucine-rich repeat genes and the finetuning of synapses. Biol. Psychiatry 69, 820-82 (2011).

108. Ko, J., Fuccillo, M. V., Malenka, R. C. \& Südhof, T. C. LRRTM2 functions as a neurexin ligand in promoting excitatory synapse formation. Neuron 64, 791-798 (2009).

109. Linhoff, M. W. et al. An unbiased expression screen for synaptogenic proteins identifies the LRRTM protein family as synaptic organizers. Neuron 61, 734-749 (2009).

110. Siddiqui, T. J., Pancaroglu, R., Kang, Y., Rooyakkers, A $\&$ Craig, A. M. LRRTMs and neuroligins bind neurexins with a differential code to cooperate in glutamate synapse development. J. Neurosci. 30, 7495-7506 (2010).

111. De Wit, J. et al. LRRTM2 interacts with Neurexin 1 and regulates excitatory synapse formation. Neuron 64 , 799-806 (2009).

112. Südhof, T. C. Neuroligins and neurexins link synaptic function to cognitive disease. Nature 455, 903-911 (2008).

113. DeLisi, L. E. et al. Hand preference and hand skill in families with schizophrenia. Laterality 7, 321-332 (2002).

114. Orr, K. G., Cannon, M., Gilvarry, C. M., Jones, P. B. \& Murray, R. M. Schizophrenic patients and their firstdegree relatives show an excess of mixed-handedness. Schizophr. Res. 39, 167-176 (1999).

115. Csernansky, J. G. et al. Abnormalities of thalamic volume and shape in schizophrenia. Am. J. Psychiatry 161, 896-902 (2004)

116. DeLisi, L. E. et al. Anomalous cerebral asymmetry and language processing in schizophrenia. Schizophr. Bull. 23, 255-271 (1997).

117. Kawasaki, Y. et al. Anomalous cerebral asymmetry in patients with schizophrenia demonstrated by voxelbased morphometry. Biol. Psychiatry 63, 793-800 (2008).

118. Oertel-Knöchel, V., Knöchel, C., Stäblein, M. \& Linden, D. E. J. Abnormal functional and structural asymmetry as biomarker for schizophrenia. Curr. Top. Med. Chem. 12, 2434-2451 (2012)

119. Shenton, M. E., Dickey, C. C., Frumin, M. \& McCarley, R. W. A review of MRI findings in schizophrenia. Schizophr. Res. 49, 1-52 (2001)

120. Sommer, I., Ramsey, N., Kahn, R., Aleman, A. \& Bouma, A. Handedness, language lateralisation and anatomical asymmetry in schizophrenia: meta-analysis. Br. J. Psychiatry J. Ment. Sci. 178, 344-351 (2001).

121. Bailey, A. et al. Autism as a strongly genetic disorder: evidence from a British twin study. Psychol. Med. 25 63-77 (1995).

122. Boles, D. B., Barth, J. M. \& Merrill, E. C. Asymmetry and performance: toward a neurodevelopmental theory. Brain Cogn. 66, 124-139 (2008)

123. De Fossé, L. et al. Language-association cortex asymmetry in autism and specific language impairment. Ann. Neurol. 56, 757-766 (2004).
124. Herbert, M. R. et al. Abnormal asymmetry in language association cortex in autism. Ann. Neurol. 52, 588-596 (2002)

125. Herbert, M. R. et al. Brain asymmetries in autism and developmental language disorder: a nested wholebrain analysis. Brain J. Neurol. 128, 213-226 (2005).

126. Deep-Soboslay, A. et al. Handedness, heritability, neurocognition and brain asymmetry in schizophrenia. Brain J. Neurol. 133, 3113-3122 (2010).

127. Scerri, T. S. et al. PCSK6 is associated with handedness in individuals with dyslexia. Hum. Mol Genet. 20, 608-614 (2011).

128. Arning, L. et al. PCSK6 VNTR polymorphism is associated with degree of handedness but not direction of handedness. PLOS ONE 8, e67251 (2013).

129. Brandler, W. M. et al. Common variants in left/right asymmetry genes and pathways are associated with relative hand skill. PLoS Genet. 9, e1003751 (2013).

130. McManus, I. C., Martin, N., Stubbings, G. F., Chung, E. M. K. \& Mitchison, H. M. Handedness and situs inversus in primary ciliary dyskinesia. Proc. Biol. Sci. 271, 2579-2582 (2004).

131. Tanaka, S., Kanzaki, R., Yoshibayashi, M., Kamiya, T. $\&$ Sugishita, M. Dichotic listening in patients with situs inversus: brain asymmetry and situs asymmetry. Neuropsychologia 37, 869-874 (1999).

132. Geschwind, N. \& Galaburda, A. M. Cerebral Lateralization (MIT Press, 1987).

133. Lust, J. M. et al. Differential effects of prenatal testosterone on lateralization of handedness and language. Neuropsychology 25, 581-589 (2011).

134. Stein, J. L. et al. Identification of common variants associated with human hippocampal and intracranial volumes. Nature Genet. 44, 552-561 (2012).

135. Guadalupe, T. et al. Measurement and genetics of human subcortical and hippocampal asymmetries in large datasets. Hum. Brain Mapp. http://dx.doi. org/10.1002/hbm.22401 (2013)

136. Button, K. S. et al. Power failure: why small sample size undermines the reliability of neuroscience. Nature Rev. Neurosci. 14, 365-376 (2013).

\section{Acknowledgements}

S.E.F. and C.F. acknowledge support from the Max Planck Society

Competing interests statement

The authors declare no competing interests.

FURTHER INFORMATION

Cognomics: http://www.cognomics.nl/big.html

ALL LINKS ARE ACTIVE IN THE ONLINE PDF 